\title{
Role of quasicylindrical waves and surface plasmon polaritons on beam shaping with resonant nanogratings in the infrared
}

\author{
C. H. Gan, ${ }^{1}$ J. R. Pugh, ${ }^{2}$ M. J. Cryan, ${ }^{2}$ J. G. Rarity, ${ }^{2}$ and G. R. Nash ${ }^{1}$ \\ ${ }^{1}$ College of Engineering, Mathematics and Physical Sciences, University of Exeter, Exeter EX4 4QF, United Kingdom \\ ${ }^{2}$ Department of Electrical and Electronic Engineering, University of Bristol, Bristol BS8 1UB, United Kingdom
}

(Received 2 April 2014; published 30 May 2014)

\begin{abstract}
The role of quasicylindrical waves and surface plasmon polaritons in beam shaping with resonant nanogratings is investigated. It is shown that the field on the grating surface can be strongly influenced by plasmons and quasicylindrical waves in the infrared. A method that combines far-field measurements with the fast Fourier transform to map the field amplitude at the grating surface is demonstrated. For samples with a small degree of geometric asymmetry, it is shown that the imaginary part of the transform (with null zeroth-order component) can better map the amplitude of the resonant surface waves than the full complex-valued transform. Our results will impact the study, design, and footprint of resonant nanogratings.
\end{abstract}

DOI: 10.1103/PhysRevB.89.201415

PACS number(s): 42.25.Fx, 42.30.Va, 42.79.Dj, 68.47.De

Advances in nanofabrication and optical near-field measurement techniques have triggered extensive studies of light-matter interactions on patterned nanostructures for manipulating the diffraction of electromagnetic fields at metallic surfaces [1-6]. In particular, surface waves supported on metallic nanogratings give rise to numerous exotic optical phenomena such as the well-known Wood's anomalies, extraordinary optical transmission (EOT), and beam shaping/steering of light radiated from subwavelength apertures [5-9]. At optical frequencies, the field scattered on metallic surfaces by subwavelength indentations is composed mainly of surface plasmon polaritons (SPPs) and a residual "direct contribution" now widely referred to as quasicylindrical waves (QCWs) [2-4,9-11]. For intermediate infrared (IR) wavelengths $(0.8 \lesssim$ $\lambda \lesssim 5 \mu \mathrm{m})$, the scattered SPP and QCW amplitudes in nanostructures are comparable [2], and it can be challenging to distinguish between their contributions. Recently, it has been shown experimentally that the QCW is responsible for half the total transmission in EOT achieved through hole arrays illuminated with near-IR light [9], highlighting the important role that QCWs can play in metallic nanogratings. Additionally, QCW properties such as the cross-conversion with the SPP [12], its relatively weak degree of spatial coherence [13], and the existence of a transient near-zone regime where its effective wavelength varies with propagation [14], have been revealed in previous investigations. In this paper, we investigate theoretically and experimentally the contribution of QCWs and SPPs to beam shaping in the infrared using resonant nanogratings, and show that beam shaping could be achieved with QCWs alone.

Figure 1(a) shows beam shaping of light radiated from a narrow slit (width $a$ ) perforated in an optically thick metal plate (thickness $t$ ). We focus on the transverse-magnetic (TM) polarization, for which surface waves can be resonantly excited by the nanogrooves when the grating equation $k_{0} \sin \theta=$ $2 m \pi / \Lambda-k_{s}$ is satisfied [15], with $k_{0}=2 \pi / \lambda, \theta$ the angle of the diffracted beam, $m$ an integer, $\Lambda$ the period of the grating, and $k_{s}$ the wave number of the surface wave. Without the grooves, the far-field radiation pattern $I^{(0)}$ resembles a broad lobe. Throughout this article, all field quantities pertaining to the case of the isolated slit are superscripted with (0). With suitably spaced nanogrooves (width $w$ and depth $h$ ) around the slit, surface waves emerging from the slit (black thick arrows) are reflected to form standing waves that decay in amplitude along the metal surface ( $\sigma$ plane), and constructive interference between the scattered fields produce a directional beam $I$.

We begin by addressing the questions: (i) Can beam shaping be achieved with QCWs alone, and (ii) is it the QCW or the SPP that contributes dominantly to the observed beaming in the IR? To answer the first question, we analyze a resonant grating structure where the SPP field is dominant and another that supports only the QCW. Such a scenario can be achieved with gold and tungsten films in the visible spectrum. At $\lambda=0.6 \mu \mathrm{m}$, SPPs can be excited efficiently on the gold film. However, the tungsten film $\left(n_{\mathrm{W}}=3.57+i 2.86\right)$, which behaves as a strongly absorbing dielectric, only supports QCWs [11]. For all simulations, the refractive index is taken from the data of Palik [16], the number of grooves (if present) on each side of the slit is taken to be $N=10$, the incident illumination is taken to be a plane wave with unity (modulus) Poynting vector, the thickness $t$ is taken to be $1 \mu \mathrm{m}$ unless otherwise specified, and the structures are simulated using a Green Tensor formalism [17]. The amplitude of the total magnetic field $H$ along the $\sigma$ plane of the nanograting (dashed curves), and the amplitude of the magnetic field components associated with the QCW $\left(H_{\mathrm{cw}}^{(0)}\right)$ and SPP $\left(H_{\mathrm{sp}}^{(0)}\right)$ scattered by the isolated slit (solid curves), are shown for the gold and tungsten film in Figs. 1(b) and 1(c). Clearly, standing waves with decaying amplitudes are sustained along the $\sigma$ plane in both cases. However, their behavior differs in several aspects. For the gold film, $|H|$ falls rapidly below $\left|H_{s p}^{(0)}\right|$ after the first couple of grooves. Whereas for the tungsten film, $|H|$ falls below $\left|H_{\mathrm{cw}}^{(0)}\right|$ only after several wavelengths in the vicinity of the fourth groove. The SPP is more strongly trapped due to its well-defined wave vector, in contrast to the mixture of wave vectors $[4,14]$ that constitute the QCW. The lower amplitude of $H$ compared to that of $H_{\mathrm{sp}}^{(0)}\left(H_{\mathrm{cw}}^{(0)}\right)$ indicates that part of the energy of the SPP (QCW) that emerges from the slit are trapped within the nanogrooves. The dominance of the SPP for the gold film can also be inferred from the steplike decay of $|H|$ between the grooves, which is typical of the piecewise-constant SPP excitation strength along 
(a)
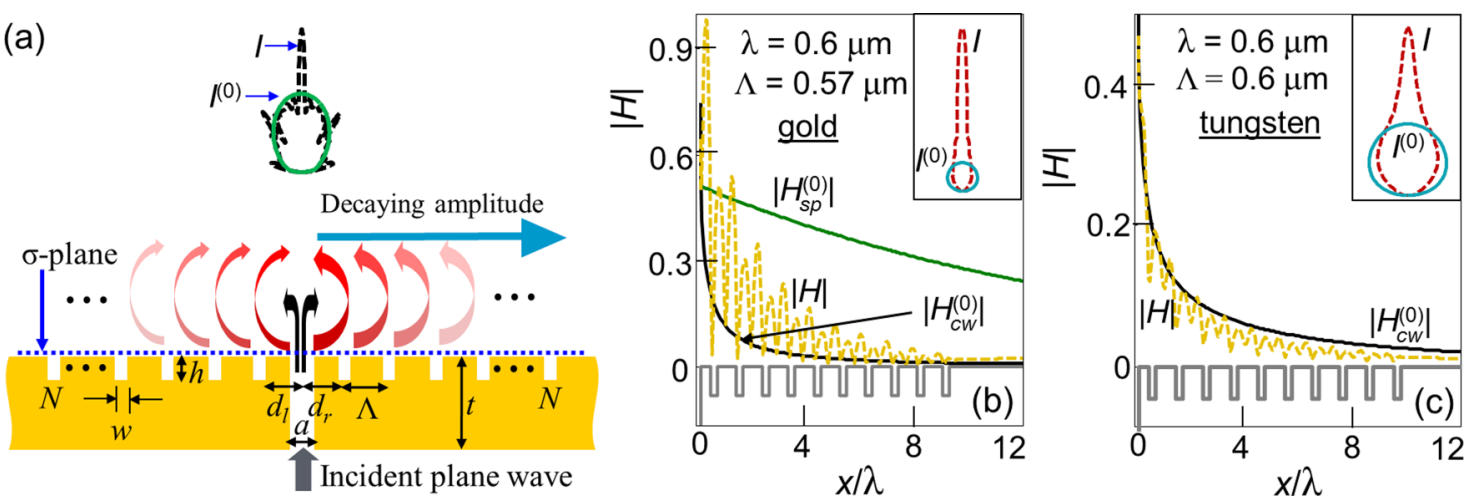

FIG. 1. (Color online) Beam shaping with a narrow slit surrounded by $2 N$ nanogrooves mediated by QCWs and SPPs. (a) At resonance, multiply reflected surface waves between the grooves form standing waves whose amplitude decreases away from the central slit. Fields on the $\sigma$ plane with (a) dominant SPPs on a gold film, and (b) QCWs alone on a tungsten film. [Insets of (b) and (c)] Radiation patterns with ( $I$ ) and without the nanogrooves $\left(I^{(0)}\right)$. In (b) and (c), it is taken that $a=200 \mathrm{~nm}, d_{l}=d_{r}=400 \mathrm{~nm}, w=150 \mathrm{~nm}, h=50 \mathrm{~nm}, \lambda=600 \mathrm{~nm}$, and the gray outline indicates the positions of the grooves.

nanogrooves [4]. The full width half maximum (FWHM) of the radiated beam [see insets of Figs. 1(b) and 1(c)] improves from $\sim 130^{\circ}$ for the isolated slit (both gold and tungsten) to about $7^{\circ}$ and $10^{\circ}$ for the gold and tungsten film, respectively [18]. From this simple example, it is apparent that (i) $Q C W s$ can be entirely responsible for the beaming of light emanated from subwavelength apertures, and (ii) strikingly different near-field distributions can result in very similar far-field beam patterns.

Next, we consider a grating structure of period $\Lambda=3 \mu \mathrm{m}$ designed to produce a forward-directed beam for $\lambda=3 \mu \mathrm{m}$ $(m=1)$ and for $\lambda=1.5 \mu \mathrm{m}(m=2)$. It is taken that $a=$ $0.9 \mu \mathrm{m}, w=0.4 \mu \mathrm{m}, h=0.35 \mu \mathrm{m}$, and $d_{l}=d_{r}=1.9 \mu \mathrm{m}$. For both orders, the wave number of the QCW and SPP is very close to the free-space wave number, i.e., $k_{\mathrm{sp}} \approx k_{\mathrm{cw}} \approx k_{0}$, and the simulated FWHM of the radiated beam pattern (not shown) is $\sim 5^{\circ}$. The amplitude of the magnetic field $H$ on the $\sigma$ plane, together with $\left|H_{\mathrm{cw}}^{(0)}\right|$ and $\left|H_{\mathrm{sp}}^{(0)}\right|$ associated with the isolated slit, are shown in Figs. 2(a) and 2(b) for $\lambda=3 \mu \mathrm{m}$ and $\lambda=1.5 \mu \mathrm{m}$, respectively. Between the slit and the first groove in both cases the standing wave amplitude decays rapidly following that of the QCW, indicating its strong influence for very short distances. From the first to the tenth groove, the amplitude of the standing waves still follows the decay of the QCW for $\lambda=3 \mu \mathrm{m}$, while the amplitude between grooves becomes more steplike for $\lambda=1.5 \mu \mathrm{m}$. This transition for
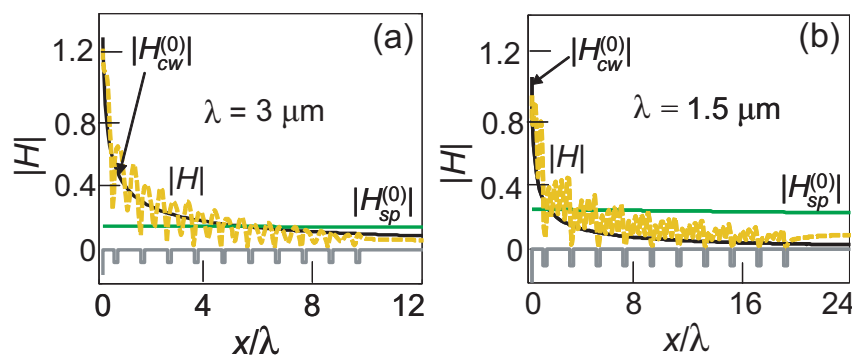

FIG. 2. (Color online) Mixed QCW and SPP contributions in IR resonant gold nanogratings $(\Lambda=3 \mu \mathrm{m})$. Scattered magnetic field components along the $\sigma$ plane for (a) $\lambda=3 \mu \mathrm{m}$ and (b) $\lambda=1.5 \mu \mathrm{m}$. $\lambda=1.5 \mu \mathrm{m}$ can be attributed to $\left|H_{\mathrm{cw}}^{(0)}\right|$ rapidly falling to lower levels than $\left|H_{\mathrm{sp}}^{(0)}\right|$ just after the first groove. Beyond the tenth groove, $|H|$ falls below both $\left|H_{\mathrm{cw}}^{(0)}\right|$ and $\left|H_{\mathrm{sp}}^{(0)}\right|$ for $\lambda=3 \mu \mathrm{m}$, suggesting that the SPP still contributes to the resonance even though the overall scattering behavior is dominated by the QCW. On the other hand for $\lambda=1.5 \mu \mathrm{m},|H|$ falls in between $\left|H_{\mathrm{cw}}^{(0)}\right|$ and $\left|H_{\mathrm{sp}}^{(0)}\right|$ beyond the tenth groove, indicating that the SPP contributes dominantly to the resonance despite a strong influence from the QCW initially. Simulations for some values of the nearest groove separations $1 \mu \mathrm{m}<d_{l}, d_{r}<3 \mu \mathrm{m}$ with all other geometrical parameters kept identical show that, apart from a marginal shift in the field amplitudes, the fields scattered on the $\sigma$ plane exhibit similar trends. For long wavelengths where metals behave like perfect conductors $(\lambda \gtrsim 6 \mu \mathrm{m})$, the QCW predominates over the SPP for propagation distances $x<20 \lambda$. Simulations for a gold film for $\lambda=6 \mu \mathrm{m}$ shows that the amplitude of the magnetic field at the $\sigma$ plane exhibits similar trends as for the case of the tungsten film [see Fig. 1(c)].

To experimentally investigate the role of the QCW and SPP, a nanograting designed for resonances at $\lambda=3 \mu \mathrm{m}$ and $\lambda=1.5 \mu \mathrm{m}$ with a nominal period $\Lambda=3 \mu \mathrm{m}$ (similar to that examined in Fig. 2) was patterned on a 1.1- $\mu$ m-thick gold layer, deposited on a calcium fluoride substrate, using a focused ion beam. A scanning electron microscopy (SEM) image of the sample, Fig. 3(a), reveals two notably asymmetric features: (i) $d_{l}=1.41 \mu \mathrm{m}$ while $d_{r}=1.28 \mu \mathrm{m}$, and (ii) the separation between the first and second nanogrooves on the left is $3.2 \mu \mathrm{m}$. The other geometric parameters are $a=$ $900 \mathrm{~nm}, w=400 \mathrm{~nm}$, and $h=350 \mathrm{~nm}$. Light emitted from a tuneable nanoLevante OPO laser in the wavelength range 1.4-4.2 $\mu \mathrm{m}$ was focused to a spot size of $\sim 10 \lambda$ at the sample [see Fig. 3(b)]. The far-field radiation pattern was measured by collecting the light scattered by the sample at different angles; the collection optics consisted of a collimating objective that directs light to the detector via a focusing parabolic mirror mounted on a translation stage. Reflecting objectives instead of refractive ones were used to minimize chromatic aberrations. The measured intensity distributions for six wavelengths ranging from $\lambda=1.5 \mu \mathrm{m}$ to $\lambda=3.8 \mu \mathrm{m}$ 
(a)
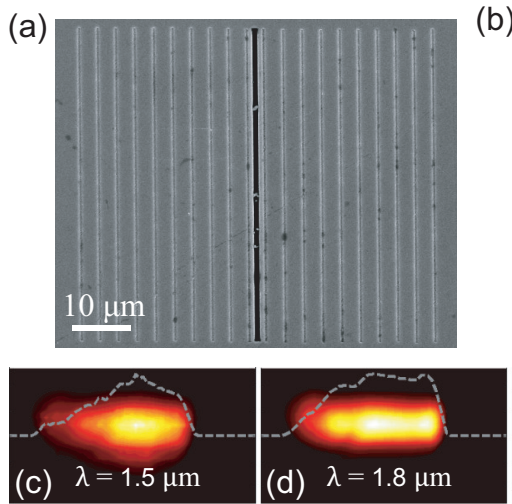

(b)

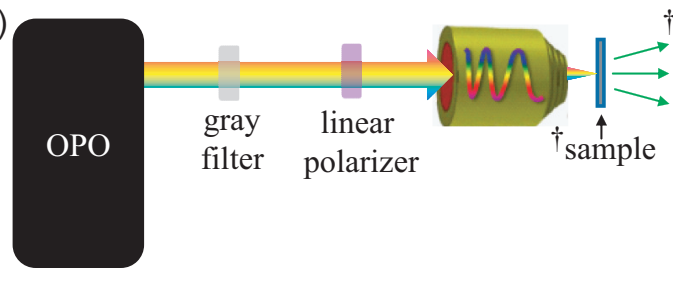

$\dagger$ Mounted on a $x y z$ translation stage

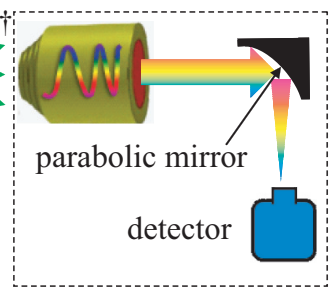

detector

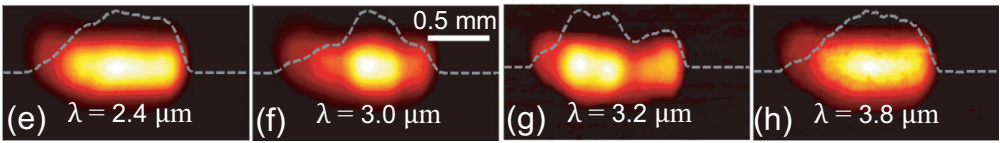

FIG. 3. (Color online) Beam shaping with a slightly asymmetric nanograting. (a) SEM image of sample. (b) Experimental setup to capture the far-field radiation pattern. Linearly polarized light along $x$ is focused onto the sample through a 36X reflecting objective (numerical aperture $\mathrm{NA}=0.5)$. Light scattered by the sample is collimated by a $15 \mathrm{X}$ reflecting objective $(\mathrm{NA}=0.3)$, which is then focused onto the single element detector. (c)-(h) Measured intensity distributions for various wavelengths. The horizontal bar scale in (f) applies to (c)-(h).

are shown in Figs. 3(c)-3(h), depicting how the beam pattern changes between on- and off-resonance. The 3-dB beamwidth of the cross section along $x$ (gray dashed lines) for the off-resonance cases (e.g., $\lambda=1.8 \mu \mathrm{m}$ and $2.4 \mu \mathrm{m}$ ) is almost double that of the narrowest beam obtained for the $m=1$ resonance at $\lambda=3 \mu \mathrm{m}$. On reducing the wavelength, the beam spreads. When the $m=2$ resonance is reached at $\lambda=1.5 \mu \mathrm{m}$, the beam can be seen to narrow again. On increasing the wavelength to $\lambda=3.2 \mu \mathrm{m}$, two distinct off-centered beams can be observed. This is due to beam steering from the nanograting, and is verified with simulation of the far-field radiation pattern with actual geometric parameters. On further increasing the wavelength to $\lambda=3.8 \mu \mathrm{m}$, beam spreading is once again observed.

The scaled Fourier transform (FT) of the measured intensity distribution $f(x, y)$ may be expressed as $F(u, v)=$ $\int_{-\infty}^{\infty} f(x, y) \exp [-i 2 \pi(x u+y v) / \lambda z] d x d y$, with $z$ the distance between the $x y$ plane and $u v$ plane [19]. For efficient computation, the fast Fourier transform (FFT) decomposition with 4096 discretization points along each axis was adopted. Since $F(u, v)$ scales with both $\lambda$ and $z$, and only $\lambda$ is known, the spatial extent of $F(u, v)$ was fitted to an optimal value $z=0.25 \mathrm{~mm}$. This reconstruction method should work well at resonance, since the fields beyond the grating would have decayed significantly, in accordance with zero padding in the FFT computation [20]. The reconstructed amplitude along the $\sigma$ plane $A_{x F}=\sqrt{\left|F^{\prime}(u, 0)\right|}$ and the simulated amplitude of the magnetic field $H$ with the actual geometric parameters for $\lambda=3 \mu \mathrm{m}$ and $\lambda=1.5 \mu \mathrm{m}$ are shown in the insets of Figs. 4(a) and 4(b) for $x>0$. Here $F^{\prime}=A_{0} F$, where $A_{0}$ is a constant determined from fitting $A_{x F}$ to $|H|$. For $\lambda \gtrsim 1 \mu \mathrm{m}$, the constant $A_{0}$ may be estimated by setting $\left[A_{x F}\right]_{x=a / 2} \approx$ $\left[\left|H_{\mathrm{cw}}^{(0)}\right|\right]_{x=a / 2}$ as the latter is typically much greater than $\left|H_{\mathrm{sp}}^{(0)}\right|$. It is seen that while $A_{x F}$ maps reasonably well the average amplitude of the standing waves, it fails to capture the standing-wave character between the nanogrooves.

By taking the imaginary part of the FFT, it is found that the secondary peaks become more visible, revealing structures that better resemble the resonant fields. For $\lambda=3 \mu \mathrm{m}$, the mapped amplitude $A_{x i}=\sqrt{\left|\operatorname{Im} F^{\prime}(u, 0)\right|}=\left(A_{x F}^{4}-A_{x r}^{4}\right)^{1 / 4}$, where $A_{x r}=\sqrt{\left|\operatorname{Re} F^{\prime}(u, 0)\right|}$, is shown in Fig. 4(a). Secondary peaks occurring near $|x|=\left\{\begin{array}{lllllll}1.6 & 6.7 & 10.4 & 17.0 & 22.6 & 26.0\end{array}\right\} \mathrm{m}$ correspond well to the positions of the grooves. The magnitude of the peaks is marginally lower than $|H|$, which is probably due to the omission of the real part. The mapped amplitude $A_{x i}$ for $\lambda=1.5 \mu \mathrm{m}$ is shown in Fig. 4(b). Secondary peaks
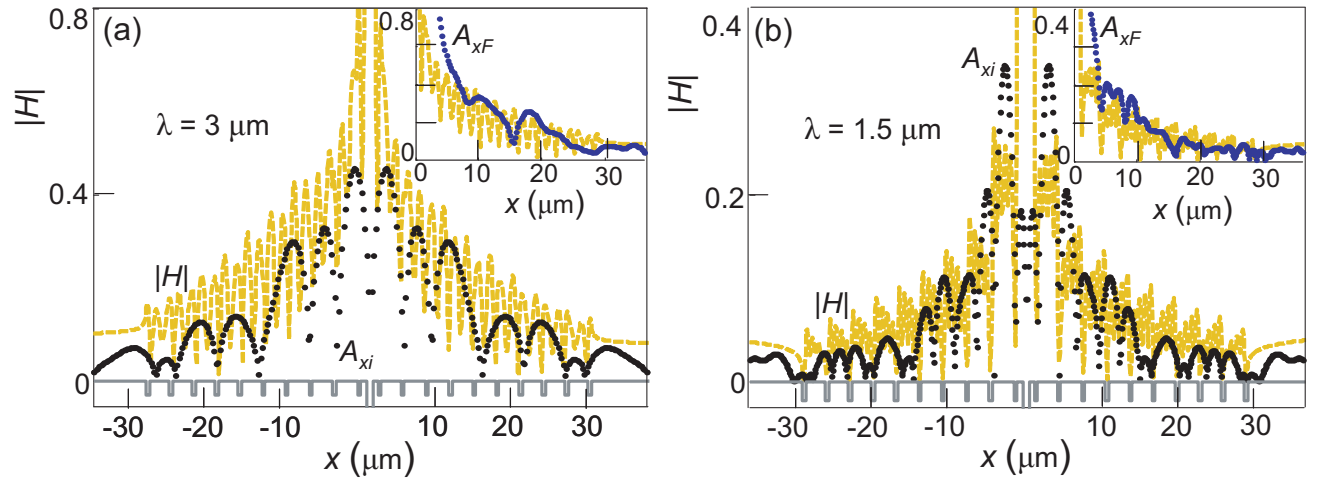

FIG. 4. (Color online) Wave-field reconstruction along the $\sigma$ plane. $A_{x i}$ superimposed on the simulated magnetic field amplitude $|H|$ for (a) $\lambda=3 \mu \mathrm{m}$, and (b) $\lambda=1.5 \mu \mathrm{m}$. [Insets of (a) and (b)] $A_{x F}$ superimposed on $|H|$ for $x>0$. 
occurring near $|x|=\left\{\begin{array}{lllllll}4.8 & 7.5 & 10.8 & 13.4 & 22.5 & 26.1 & 29.0\end{array}\right\} \mu \mathrm{m}$ correspond well again to the positions of the grooves in the actual grating. The magnitude of each of these peaks also agrees well with the simulated magnetic field amplitude $|H|$. In this case, the amplitude $A_{x r}$ (not shown) misses the standing-wave character in the vicinity of the first groove (as with $A_{x F}$ ), and spurious oscillations beyond the last groove for $x \gtrsim 30 \mu \mathrm{m}$ are present. It is remarkable that despite losing half the information, the standing-wave character of the surface waves can be mapped reasonably accurately with $\operatorname{Im} F$ alone. This improvement can be attributed to the fact that $\operatorname{Im} F$ is naturally uncluttered from the strong dc bias of the FFT, which is physically equivalent to masking off the light radiated from the central slit.

We have investigated the role of QCWs and SPPs on beam shaping in resonant metallic nanogratings. Our analysis shows that, while beam shaping can be achieved through predominant QCW or SPP contributions, both of these surface waves can play a significant part in the intermediate IR spectrum $0.8 \lesssim \lambda \lesssim 5 \mu \mathrm{m}$. We have also demonstrated how the amplitude of the surface waves along the $\sigma$ plane can be recovered by first measuring the far-field pattern followed by a Fourier transform, and how taking its imaginary part for an asymmetrical grating leads to a more accurate reconstruction. This allows a better comparison of the experimental data with simulations, defining a new method to study the underlying physics of QCWs and SPPs in these structures, and enabling the design of dielectric rather than metallic beam-shaping nanogratings.

This work was supported by the Engineering and Physical Sciences Research Council (Grant No. EP/J011932/1).
[1] G. Gay, O. Alloschery, B. Viaris de Lesegno, C. O’Dwyer, J. Weiner, and H. J. Lezec, Nature Phys. 2, 262 (2006).

[2] P. Lalanne and J. P. Hugonin, Nature Phys. 2, 551 (2006).

[3] L. Aigouy, P. Lalanne, J. P. Hugonin, G. Julié, V. Mathet, and M. Mortier, Phys. Rev. Lett. 98, 153902 (2007).

[4] P. Lalanne, J. P. Hugonin, H. Liu, and B. Wang, Surf. Sci. Rep. 64, 453 (2009).

[5] H. J. Lezec, A. Degiron, E. Devaux, R. A. Linke, L. MartínMoreno, F. J. García-Vidal, and T. W. Ebbesen, Science 297, 820 (2002).

[6] N. Yu, Q. J. Wang, and F. Capasso, Lasers Photon. Rev. 6, 24 (2012).

[7] R. W. Wood, Philos. Mag. 4, 396 (1902).

[8] T. W. Ebbesen, H. J. Lezec, H. F. Ghaemi, T. Thio, and P. A. Wolff, Nature (London) 391, 667 (1998).

[9] F. van Beijnum, C. Rétif, C. B. Smiet, H. Liu, P. Lalanne, and M. P. van Exter, Nature (London) 492, 411 (2012).

[10] W. Dai and C. M. Soukoulis, Phys. Rev. B 80, 155407 (2009).

[11] C. H. Gan, L. Lalouat, P. Lalanne, and L. Aigouy, Phys. Rev. B 83, 085422 (2011).
[12] X. Y. Yang, H. T. Liu, and P. Lalanne, Phys. Rev. Lett. 102, 153903 (2009)

[13] C. H. Gan and G. R. Nash, Opt. Lett. 38, 4453 (2013).

[14] G. Lévêque, O. J. F. Martin, and J. Weiner, Phys. Rev. B 76, 155418 (2007).

[15] H. Raether, Surface Plasmons on Smooth and Rough Surfaces and on Gratings (Springer, Berlin, 1988).

[16] E. D. Palik, Handbook of Optical Constants of Solids (Academic Press, New York, 1985).

[17] C. H. Gan and G. Gbur, Plasmonics 3, 111 (2008).

[18] Calculations yield that the radiated intensity for the tungsten film is an order of magnitude lower than for the gold film. This is due mainly to the higher dissipation loss of the fundamental mode in the slit.

[19] J. W. Goodman, Introduction to Fourier Optics, 3rd ed. (Roberts \& Company Publishers, Greenwood Village, 2005).

[20] Off resonance, the fields beyond the $N^{\text {th }}$ groove may not have decayed significantly, thus degrading the accuracy of the method. 